\title{
Comparative proteomic analysis of virulent and avirulent strains of Toxoplasma gondii reveals strain-specific patterns
}

\author{
Dong-Hui Zhou ${ }^{1, *}$, Ze-Xiang Wang ${ }^{1, *}$, Chun-Xue Zhou ${ }^{1,3}$, Shuai He ${ }^{1,4}$, Hany M. \\ Elsheikha ${ }^{2}$ and Xing-Quan Zhu ${ }^{1}$ \\ ${ }^{1}$ State Key Laboratory of Veterinary Etiological Biology, Key Laboratory of Veterinary Parasitology of Gansu Province, Lanzhou \\ Veterinary Research Institute, Chinese Academy of Agricultural Sciences, Lanzhou, Gansu Province, 730046, PR China \\ ${ }^{2}$ Faculty of Medicine and Health Sciences, School of Veterinary Medicine and Science, University of Nottingham, Sutton \\ Bonington Campus, Loughborough, LE12 5RD, UK \\ ${ }^{3}$ Department of Parasitology, Shandong University School of Basic Medicine, Jinan, Shandong Province, 250012, PR China \\ ${ }^{4}$ College of Animal Science and Technology, Anhui Agricultural University, Hefei, Anhui Province, 230036, PR China \\ *These authors contributed equally to this work \\ Correspondence to: Hany M. Elsheikha, email: hany.elsheikha@nottingham.ac.uk \\ Xing-Quan Zhu, email: xingquanzhu1@hotmail.com
}

Keywords: Toxoplasma gondii, oocyst, proteomics, iTRAQ, differentially expressed protein (DEP)

Received: April 18, $2017 \quad$ Accepted: June 18, $2017 \quad$ Published: July 07, 2017

Copyright: Zhou et al. This is an open-access article distributed under the terms of the Creative Commons Attribution License 3.0 (CC BY 3.0 ), which permits unrestricted use, distribution, and reproduction in any medium, provided the original author and source are credited.

\section{ABSTRACT}

Research exploring the proteome of Toxoplasma gondii oocysts has gained momentum over the past few years. However, little is known about the oocyst's protein repertoires that contribute to differential virulence among $T$. gondii strains. Here, we used isobaric tag for relative and absolute quantitation-based proteomic analysis of oocysts of two T. gondii strains exhibiting the virulent PYS (ToxoDB\#9) phenotype versus the less virulent PRU (Type II, ToxoDB\#1) phenotype. Our aim was to determine protein expression patterns that contribute to the virulence of a particular phenotype. A total of 2,551 proteins were identified, of which 374 were differentially expressed proteins (DEPs) ( $\mid \log _{2}$ fold change $\mid \geq 0.58$ and $P<0.05$ ). DEPs included 192 increased and 182 decreased proteins. Gene Ontology and KEGG pathway analyses revealed a large number of DEPs enriched in various metabolic processes. Protein interaction network analysis using STRING identified inosine monophosphate dehydrogenase (IMPDH), Bifunctional GMP synthase/glutamine amidotransferase protein, Glucose-6-phosphate 1-dehydrogenase, and Citrate synthase as the top four hubs. Of the $\mathbf{2 2}$ virulence proteins commonly expressed in the oocysts of the two strains, 13 and 2 proteins were increased in PYS strain and PRU strain, respectively. Also, 10 and 3 of the 22 identified oocyst wall proteins showed higher expression in oocysts of PRU strain and PYS strain, respectively. These findings revealed new proteomic differences in the oocysts of $T$. gondii strains of different genotypic backgrounds.

\section{INTRODUCTION}

The apicomplexan protozoan parasite Toxoplasma gondii has a remarkable ability to infect a wide range of mammals, birds and humans [1-4]. Its indirect lifecycle involves asexual phase in the intermediate vertebrate host and sexual phase that occurs exclusively in the intestinal epithelium of the definitive felid host. T. gondii undergoes stage transformation during its development from tachyzoite to bradyzoite in the intermediate host to oocyst's stage in the feline definitive host. Infection can occur via ingestion of the cat-borne oocysts through contaminated food or water, ingestion of cysts in the tissues of an infected intermediate host or transplacentally from infected mothers to their offspring [5]. The ability of the different forms of the parasite's life cycle, in 
particular the oocyst stage, to adapt to and interact with various niches inside and outside the host is essential for successful colonization and infection [6]. Oocysts have an exceptional ability to adapt to adverse environmental conditions outside the host [7]. They can remain viable for at least 1 year at $4^{\circ} \mathrm{C}$ [8]. Oocysts remained viable for at least 4.5 years in fresh water and 2 years in marine water [9]. Exposure to ultraviolet irradiation at doses even exceeding those used to treat water did not fully inactivate $T$. gondii oocysts [10]. Sodium hypochlorite and ozone, two chemicals commonly used to treat sewage and drinking water, failed to inactivate all infective oocysts [11]. Interestingly, clinical illness can be more severe when hosts acquire the infection through ingestion of oocysts [12].

The clinical consequence of $T$. gondii infection in human ranges from asymptomatic to debilitating or even life threatening. There are likely many reasons for these differences. T. gondii displays a high degree of genetic diversity and includes strains with distinct virulence potentials. Previous studies identified three clonal lineages of $T$. gondii that exhibit different virulence phenotypes in mice, with type I strains are lethal in mice, but type II and III strains are less virulent [13-14]. In addition to the parasite genotype and virulence [13-16], the age and immune status of the infected host [4], and stage of the life cycle causing infection [12] were all found to influence the severity of toxoplasmosis. Also, the pathogenicity of $T$. gondii strains has been found to be correlated with their migratory capacity, the growth rate in vitro and the parasite loads in mice [15], and polymorphism and differential expression of the dense granule and rhoptry proteins [16]. Despite significant progress made in recent years in understanding the molecular basis of different pathogenic behaviors of distinct $T$. gondii genotypes, the mechanisms that underpin differences in the outcome of infection are not completely known.

In recent years, interests in $T$. gondii oocysts have increased due to their important role in the transmission of T. gondii to new hosts and ecosystems [17], and because of their links to increased infections in marine mammals and water-borne outbreaks in humans [18]. Transcriptomic and proteomic studies addressing the molecular structure of oocysts have been reported [19-22]. Proteins uniquely expressed in the oocysts have been identified [19-21] and proteins that mediate the adaptation of oocysts to nutrient-poor and stressing extracellular environment have been discovered [23]. Additionally, proteomic changes that occur during oocyst sporulation have been revealed [24-25]. However, knowledge of the differentially expressed proteins that promote virulence between strains of different genotypes is still limited. The availability of phenotypically distinct strains - virulent type I strain and less virulent type II strain of $T$. gondii - provides the opportunity for identifying strain-specific virulence factors. In the present study, we compared oocyst's protein expression of the virulent ToxoDB\#9 (PYS) strain and the less virulent ToxoDB\#1 (PRU) strain using iTRAQ (isobaric tag for relative and absolute quantitation)-based LC-MS/MS analysis. Our results revealed genotypespecific differences in the proteomes of oocysts of the two genotypic variants of $T$. gondii oocysts.

\section{RESULTS AND DISCUSSION}

The aim of the study was to compare the proteomic profiles of virulent PYS strain with avirulent PRU T. gondii strain using quantitative iTRAQ-based proteomic and bioinformatic analyses. We tested whether there are phenotype-specific differentially expressed proteins that promote virulence and/or tolerance of the oocysts to environmental conditions. A total of 2,551 proteins were identified, of which 374 were differentially expressed proteins (DEPs) between the two parasite strains. Interestingly, about half of the GO terms under biological process and 12 pathways in the top 27 enriched KEGG pathways were related to metabolic activities, underscoring the roles of metabolism in the observed differences between the two parasite strains.

\section{Identification and expression of proteins}

A total of 404,046 spectra were obtained, of which 86,777 spectra were identified using MASCOT Server 2.4.0 (Matrix Science). Also, 51,728 distinct peptides were identified and 2,551 proteins were detected after grouping with unused score $\geq 1.3$ and confidence value $>95 \%$. The variation coefficients between the two biological replicates covered $99 \%$ of the identified proteins when the coefficient of variation $(\mathrm{CV})<50 \%$ (Figure 1 ). The results of hierarchical clustering analysis of the two biological replicates (each contained two pooled technical replicates) are shown in Figure 2. Cluster of orthologous group (COG) analysis divided the identified proteins into 26 categories. As shown in Figure 3, translation, ribosomal structure and biogenesis; posttranslational modification, protein turnover and chaperones; general function prediction only; signal transduction mechanisms; and energy production and conversion were the five categories with the most identified proteins.

DEPs were identified by comparing the relative protein expression values between PYS strain and PRU strain. A total of 374 proteins were classified as DEPs ( $\mid \log _{2}$ fold change $\mid \geq 0.58$ and $P<0.05$ ), of which 192 were increased and 182 were decreased (Supplementary Table 1 and Supplementary Table 2). GTP-binding protein lepA (TGME49_307980), Toxoplasma gondii family D protein (TGME49_271590), TGME49_294600, TGME49_205090, and toxofilin (TGME49_214080) were the top five increased proteins. By contrast, the top five mostly decreased proteins were dense granule protein GRA7 (TGME49_203310), TGME49_203890, rhoptry 
protein ROP5 (TGME49_308090), Zn-containing alcohol dehydrogenase (TGME49_311780), and nudix-type motif 9 isoform a family protein (TGME49_247220).

\section{Functional annotation of DEPs}

To elucidate the biological roles of the DEPs between oocysts of PYS and PRU strains, GO enrichment analysis using GOseq R package was performed. The differentially increased and decreased proteins were classified into three GO categories, namely biological process, cellular component and molecular function. The top 10 most abundant GO terms in the three categories are presented for increased and decreased proteins (Figure 4A-4B). The top five enriched GO terms for increased proteins under biological process included small molecule metabolic process, cellular protein modification process, biosynthetic process, cellular nitrogen compound metabolic process, and translation. By contrast, cellular protein modification process, cellular nitrogen compound metabolic process, biosynthetic process, carbohydrate metabolic process, and small molecule metabolic process were the top five abundantly enriched terms for the decreased proteins.

The top three increased GO terms of the cellular component category included intracellular, cytoplasm and protein complex, whereas the top three decreased terms included extracellular region, cytoplasm and cell. In the category of molecular function, ion binding was the most significantly enriched GO term. KEGG pathway enrichment analysis revealed 164 DEPs, which had a KEGG Orthology (KO) ID and were related to 51 pathways. The 27 highly enriched pathways are represented in Figure 5. The 10 most significantly enriched of which were metabolic pathways, biosynthesis of secondary metabolites, biosynthesis of antibiotics, carbon metabolism, biosynthesis of amino acids, Citrate cycle (TCA cycle), peroxisome, ribosome, propanoate metabolism, and valine, leucine and isoleucine degradation.

\section{Differences in the expression of virulence factors}

A number of virulence factors of $T$. gondii oocysts have been shown to be increased during sporulation and these have been linked to the infectivity of sporulated oocysts [25]. In our study, we detected differences in the expression levels of these virulence proteins between sporulated oocysts of PYS and PRU strains. Among the 22 virulence proteins commonly expressed in the sporulated oocysts of PYS strain and PRU strain, only serine-threonine phosphatase 2C (PP2C) and rhoptry protein ROP16 (ROP16) had a higher expression level in the PRU strain. By contrast, 13 virulence factors, including dense granule protein GRA6 (GRA6), rhoptry neck protein RON4 (RON4), microneme protein MIC3 (MIC3), rhoptry protein ROP2A (ROP2A), dense granule protein GRA1 (GRA1), rhoptry neck protein RON5 (RON5), rhoptry protein ROP18 (ROP18), microneme protein MIC2 (MIC2), apical membrane antigen AMA1, microneme protein MIC4 (MIC4), microneme protein MIC6 (MIC6), rhoptry protein ROP5 (ROP5), and dense granule protein GRA7 (GRA7) were upregulated in the PYS strain. Furthermore, the level of expression of the following seven virulence proteins did not show any significant difference between the two strains: rhomboid protease ROM4 (ROM4), V-type $\mathrm{H}(+)$-translocating pyrophosphatase (VP1), sporozoite protein with an altered

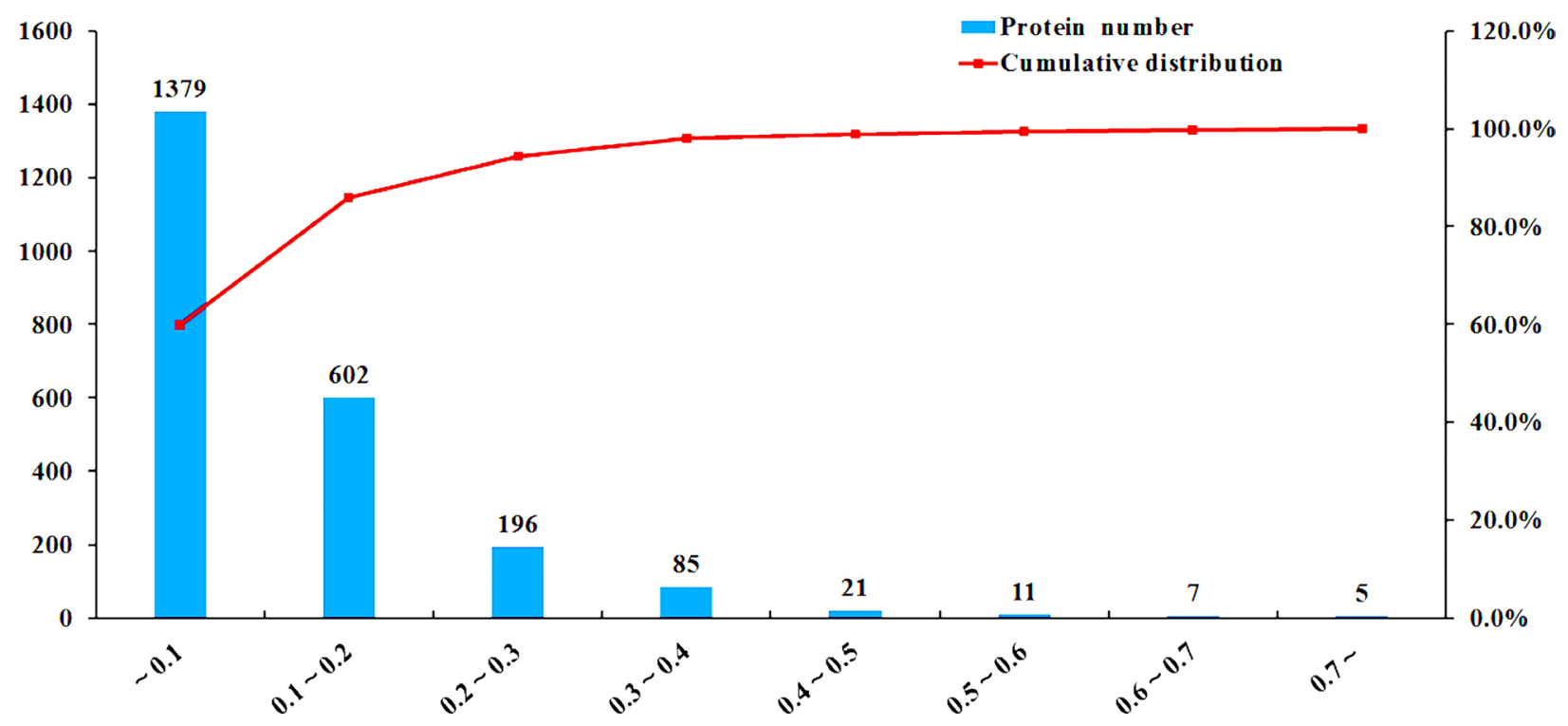

Figure 1: iTRAQ coupled with LC-MS/MS analysis of sporulated oocysts of ToxoDB\#9 (PYS) strain and ToxoDB\#1 (PRU) strain. The $\mathrm{x}$-axis represents coefficient of variation $(\mathrm{CV})$ values. The left y-axis represents the number of proteins and the right $\mathrm{y}$-axis represents the cumulative percentage of proteins (red lines). 
thrombospondin repeat (SPATR), microneme protein MIC1 (MIC1), photosensitized INA-labeled protein PHIL1 (PHIL1), microneme protein MIC8 (MIC8), and rhoptry neck protein RON2 (RON2) (Figure 6). These results indicate that sporulated oocysts of the virulent PYS strain express more virulence factors than sporulated oocysts of the less virulent PRU strain.

\section{Expression of oocyst wall proteins}

Oocyst wall proteins play important roles in the environmental tolerance of $T$. gondii oocysts and can protect the sporozoites from adverse conditions outside in the environment and inside the host [24]. The protein profile of $T$. gondii oocyst wall has already been identified

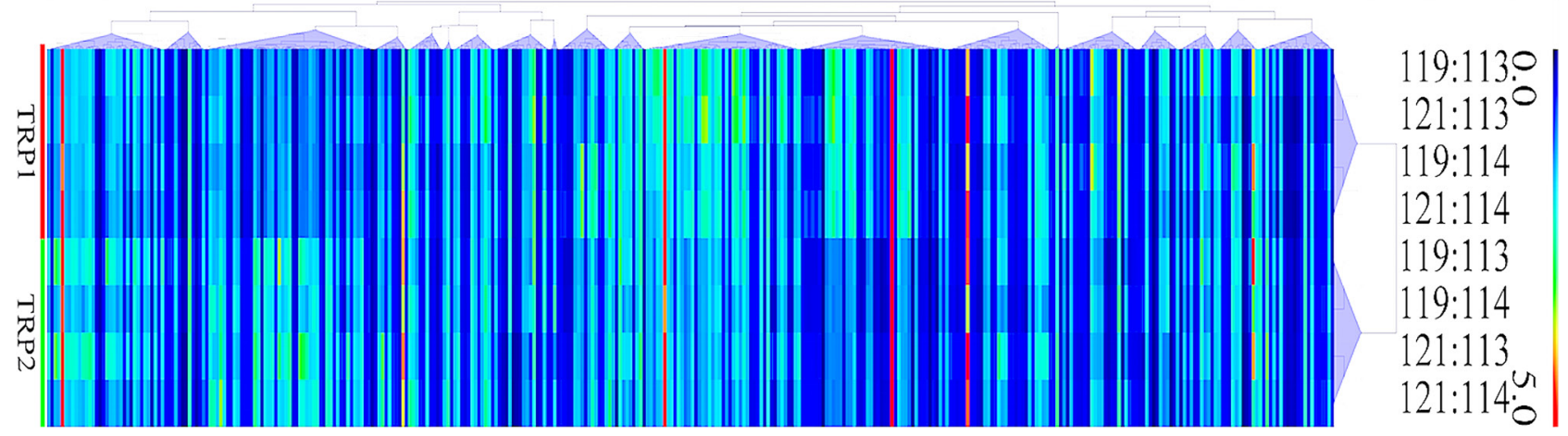

Figure 2: Hierarchical clustering of the relative abundance of oocyst's proteins of PYS strain versus PRU strain. Each column represents one protein. Rows represent the pooled samples of the two technical replicates, TRP1 and TRP2. The expression levels of the proteins are shown in red (increase) and blue (decrease) colors.

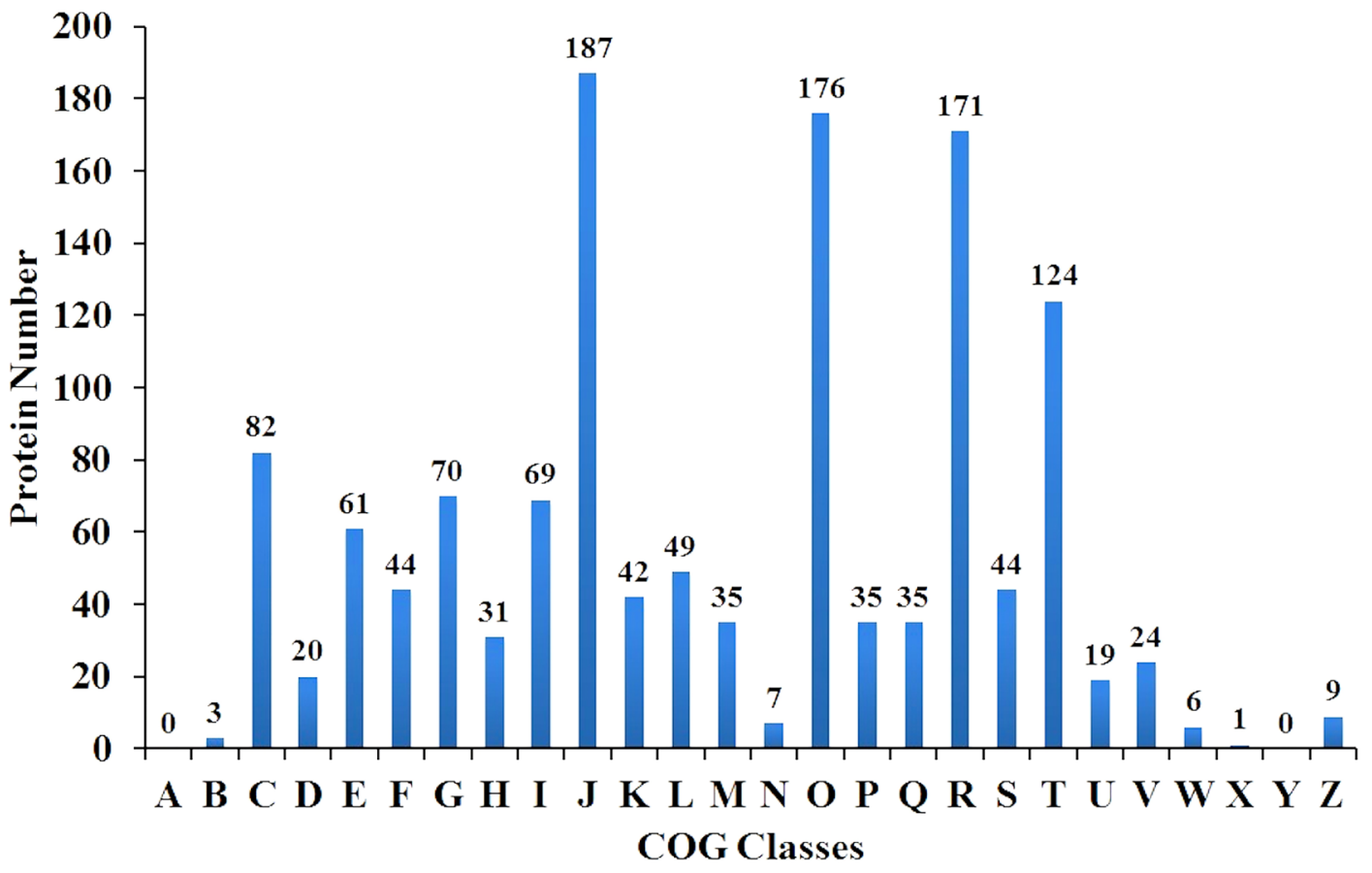

Figure 3: Distribution of cluster of orthologous groups (COG) analysis of the indentified proteins. The y-axis represents the number of proteins. The $\mathrm{x}$-axis represents different COG classes. A to Z represent: RNA processing and modification; Chromatin structure and dynamics; Energy production and conversion; Cell cycle control, cell division, chromosome partitioning; Amino acid transport and metabolism; Nucleotide transport and metabolism; Carbohydrate transport and metabolism; Coenzyme transport and metabolism; Lipid transport and metabolism; Translation, ribosomal structure and biogenesis; Transcription; Replication, recombination and repair; Cell wall/membrane/envelope biogenesis; Cell motility; Posttranslational modification, protein turnover, chaperones; Inorganic ion transport and metabolism; Secondary metabolites biosynthesis, transport and catabolism; General function prediction only; Function unknown; Signal transduction mechanisms; Intracellular trafficking, secretion, and vesicular transport; Defense mechanisms; Extracellular structures; Mobilome: prophages, transposons; Nuclear structure; Cytoskeleton, respectively. 
Table 1: Differential expression of oocyst wall proteins of Toxoplasma gondii

\begin{tabular}{|c|c|c|c|c|}
\hline Gene ID & Protein Description & CV & AVE & Differential Expression \\
\hline TGME49_235315 & PAN domain-containing protein & 0.0645 & 2.4260 & Up \\
\hline TGME49_209610 & oocyst wall protein OWP2 (OWP2) & 0.1685 & 2.4252 & $\mathrm{Up}$ \\
\hline TGME49_286250 & hypothetical protein & 0.1774 & 2.2303 & Up \\
\hline TGME49_232170 & hypothetical protein & 0.1143 & 2.2166 & Up \\
\hline TGME49_235390 & PAN domain-containing protein & 0.0756 & 1.8167 & Up \\
\hline TGME49_209470 & hypothetical protein & 0.0584 & 1.7690 & Up \\
\hline TGME49_320530 & hypothetical protein & 0.3891 & 1.6981 & Up \\
\hline TGME49_281590 & hypothetical protein & 0.1065 & 1.6710 & Up \\
\hline TGME49_253150 & hypothetical protein & 0.1386 & 1.5380 & Up \\
\hline TGME49_294820 & type I fatty acid synthase & 0.1292 & 1.5107 & Up \\
\hline TGME49_316550 & hypothetical protein & 0.1300 & 1.2186 & $\mathrm{~N}$ \\
\hline TGME49_268310 & oocyst wall protein OWP3 (OWP3) & 0.0640 & 1.1597 & $\mathrm{~N}$ \\
\hline TGME49_269380 & hypothetical protein & 0.1047 & 1.1517 & $\mathrm{~N}$ \\
\hline TGME49_248810 & nuclear factor NF7 & 0.1110 & 1.1005 & $\mathrm{~N}$ \\
\hline TGME49_254430 & microneme protein & 0.0239 & 0.9752 & $\mathrm{~N}$ \\
\hline TGME49_204420 & oocyst wall protein OWP1 (OWP1) & 0.0374 & 0.9513 & $\mathrm{~N}$ \\
\hline TGME49_203500 & $\begin{array}{l}\text { Alanine dehydrogenase/pyridine nucleotide } \\
\text { transhydrogenase domain-containing protein }\end{array}$ & 0.1033 & 0.9247 & $\mathrm{~N}$ \\
\hline TGME49_237080 & hypothetical protein & 0.2250 & 0.8431 & $\mathrm{~N}$ \\
\hline TGME49_258910 & hypothetical protein & 0.1331 & 0.5933 & Down \\
\hline TGME49_319890 & hypothetical protein & 0.2363 & 0.5931 & Down \\
\hline TGME49_306050 & hypothetical protein & 0.1883 & 0.5412 & Down \\
\hline TGME49_287250 & hypothetical protein & 0.5441 & 0.5079 & $\mathrm{~N}$ \\
\hline
\end{tabular}

Abbreviations: CV: coefficient of variation; AVE: average expression value of two biological replicates; Up: increased; Down: decreased; N: non-significant change.

[27-28]. However, in the present study we identified 22 oocyst wall proteins and analyzed their differential expression between two distinct genotypic variants of T. gondii oocysts. As shown in Table 1, 10 oocyst wall proteins were increased and three were decreased. Roughly half of the oocyst wall proteins had higher expression level in PRU oocysts compared with PYS oocysts. Only three oocyst wall proteins were overexpressed in PYS oocysts compared with PRU oocysts. The other nine oocyst proteins showed no significant difference between the two parasite strains. These discrepancies in the expression of oocyst wall proteins are likely related to differences in the ability of oocysts of PYS strain and PRU strain to survive in the environment. 


\section{Protein-protein network construction}

The protein-protein interaction (PPI) (combined score $>0.9$ ) networks of the DEPs were built using
Cytoscape software (Figure 7). The interaction network included 102 nodes and 271 edges. The degrees of interaction were defined to determine the number of neighbors that a node is directly linked to, and nodes with a
Biological process
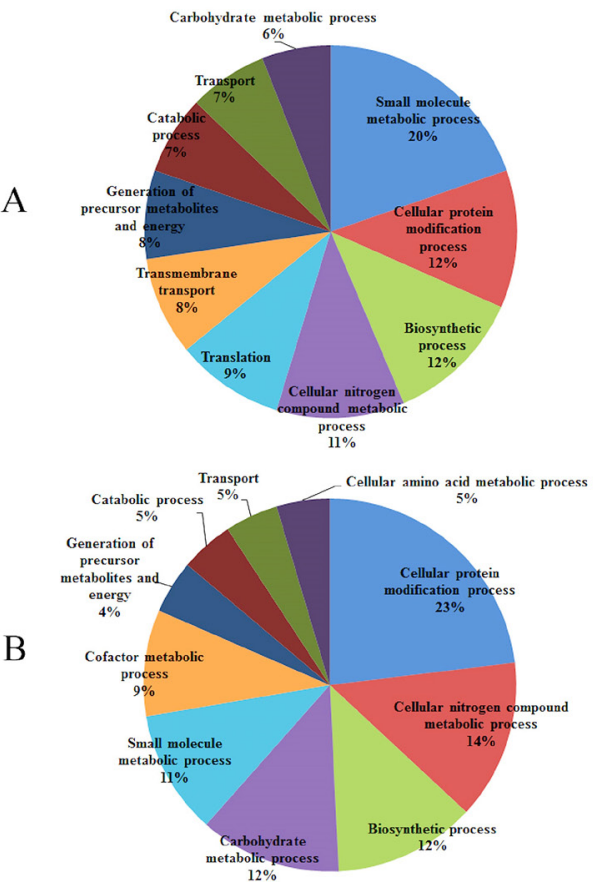

Cellular component
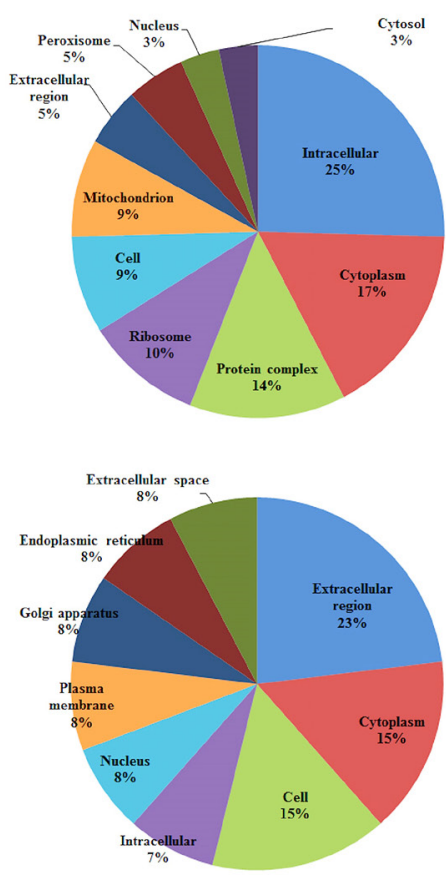

Molecular function
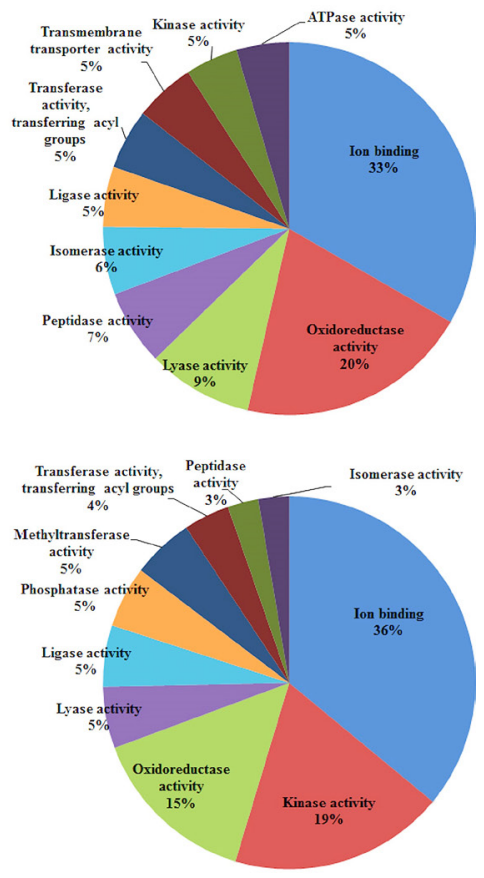

Figure 4: Gene Ontology (GO) distribution of the differentially expressed proteins (DEPs). The top 10 enriched GO terms under Biological Process, Cellular Component and Molecular Function for (A) increased proteins and (B) decreased proteins.

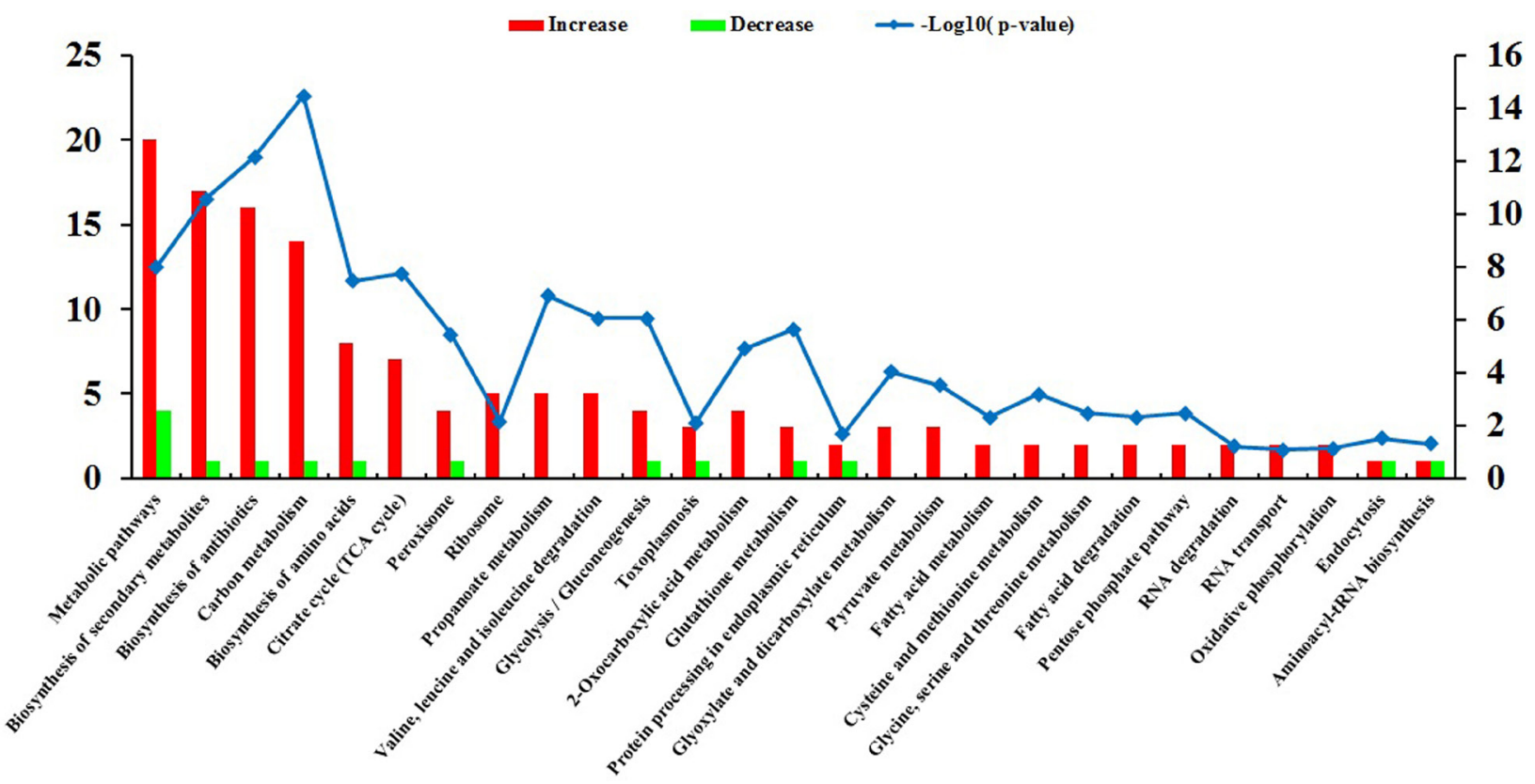

Figure 5: KEGG pathway analysis of the differentially expressed proteins (DEPs). The horizontal axis represents the significantly enriched KEGG pathways. The left y-axis represents the number of DEPs and the right y-axis represents logarithmic corrected $p$-values for significant overrepresentation $[-\log 10(p-$ value $)]$. 
high degree of interaction with other proteins were defined as 'hub' proteins. The significant hub proteins were inosine monophosphate dehydrogenase (IMPDH), Bifunctional GMP synthase/glutamine amidotransferase protein (GMP synthase), Glucose-6-phosphate 1-dehydrogenase (G6PD), and Citrate synthase (CS). All these protein hubs are known to play important roles in various metabolic processes. For example, IMPDH and GMP synthase are key enzymes in the synthesis of guanine nucleotides and have been considered good therapeutic targets [29-30]. Likewise, G6PD and the mitochondrial CS play a role in glycolysis and carbohydrate metabolism. The results of qPCR validation of these four hub proteins were consistent with the proteomic data (Figure 8). The expression level of G6PD and GMP were lower and the expression level of IMPDH and CS were higher in the PYS strain compared to the PRU strain.

In conclusion, we employed iTRAQ-based proteomic approach to profile the proteomic differences in $T$. gondii oocysts between the virulent PYS strain and the less virulent PRU strain. A total of 2,551 proteins were identified, of which 374 were differentially expressed. KEGG pathway enrichment analysis revealed that the most enriched pathways were related to metabolism and bioenergetics. While PYS oocysts showed significant increase in the expression of virulence-related proteins compared to PRU oocysts, oocyst wall proteins were highly expressed in PRU oocysts compared with PYS oocysts. Further research is needed to determine how these differentially expressed proteins contribute to the virulence of PYS strain and the environmental endurance of PRU strain. These findings contribute to our understanding of the virulence determinants of $T$. gondii strains of different genotypes and may facilitate the identification of new therapeutic targets for prevention and control of toxoplasmosis.

\section{MATERIALS AND METHODS}

\section{Ethics statement}

All animal protocols were reviewed and approved by the Animal Administration and Ethics Committee of Lanzhou Veterinary Research Institute, Chinese Academy of Agricultural Sciences. Animals were purchased from Laboratory Animal Center of Lanzhou Veterinary Research Institute. The study was performed in strict accordance with the recommendations set forth in the Animal Ethics Procedures and Guidelines of the People's Republic of China. All efforts were made to minimize animal suffering and to reduce the number of animals used to produce $T$. gondii parasites.

\section{Production and purification of sporulated oocysts}

The virulent PYS (ToxoDB\#9 genotype) and less virulent Prugniuad (PRU; ToxoDB \#1) strains were used in the present study because ToxoDB\#9 (Chinese 1) is the predominant $T$. gondii genotype in Asia and T. gondii type

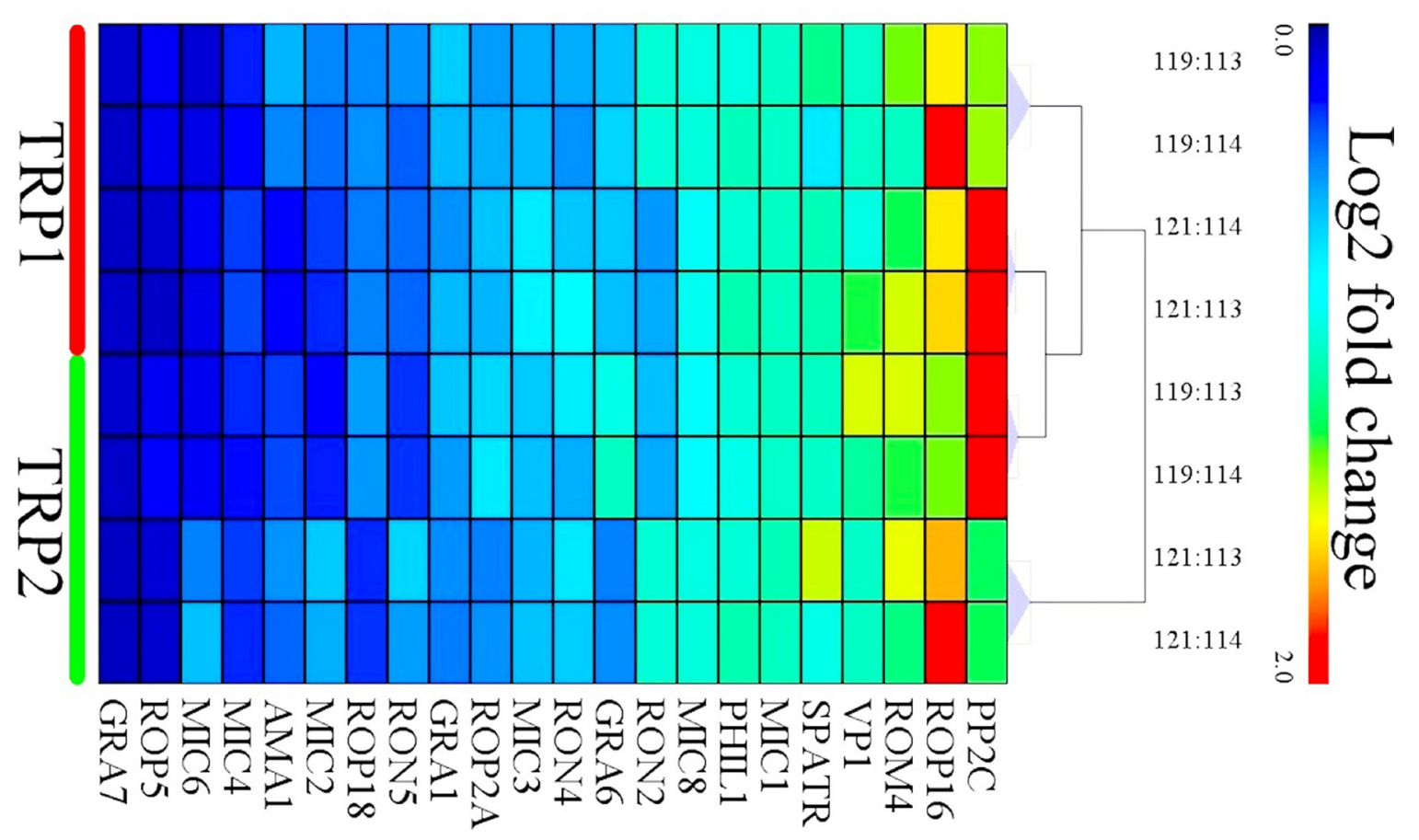

Figure 6: Heat map clustering of the intensities of the differentially expressed (DE) virulence proteins of oocysts between PYS strain and PRU strain. Expression values were $\log _{2}$-transformed and were annotated based on a gradient color scheme. The $\mathrm{x}$-axis represents the logogram of virulence proteins. The TRP1 and TRP2 in y-axis represent technical replicates 1 and 2, respectively. 
II is the most prevalent genotype in cats. Both strains are routinely maintained in our laboratory. Briefly, 16-weekold female guinea pigs were intraperitoneally inoculated with $10^{5}$ tachyzoites of PYS strain. One month after the inoculation, guinea pigs showed lethargy, loss of appetite, panting, and diarrhea. Then, animals were euthanized and the cysts in their brain and muscle tissues were collected and used to infect a specific-pathogen-free kitten. Feces from the infected kitten was collected daily and examined by zinc sulfate double centrifugation to detect the excreted oocysts. The kitten was tested by indirect fluorescentantibody test prior to infection to ensure that they were seronegative for $T$. gondii antibodies. The PRU strain was maintained via oral passage of cysts in six-week-old, female BALB/c mice. One week postinfection, infected mice exhibited reduced appetite, ruffled fur and head tilting. Specific-pathogen-free, 10-week-old, kitten was orally inoculated with 200 cysts and its feces was collected daily and examined as described above to detect oocysts of the PRU strain.

Oocysts of PYS and PRU strains were isolated from the cat feces by filtering fecal suspension through a tea strainer $(250-\mu \mathrm{m}$ pore size). Then, the fecal filtrates were pelleted by centrifugation at $60 \mathrm{~g}$ for $15 \mathrm{~min}$. The supernatant was discarded and the pellet was washed $3 \times$ with phosphate-buffered saline (PBS). After discarding the supernatant, the pellet was suspended in sucrose solution to concentrate the oocysts. Following sucrose flotation, a cesium chloride $(\mathrm{CsCl})$ density gradient method was used to purify $T$. gondii oocysts as described previously [26]. To induce sporulation, the purified oocysts were mixed with $2 \% \mathrm{H}_{2} \mathrm{SO}_{4}$ and were aerated on a shaker at ambient temperature for 7 days. Then, sporulated oocyst were washed twice with $0.85 \%$ saline, mixed with $2 \% \mathrm{H}_{2} \mathrm{SO}_{4}$ and stored at $4{ }^{\circ} \mathrm{C}$ until analysis. Prior to use, oocysts were washed $3 \times$ in PBS to remove sulfuric acid and restore neutral $\mathrm{pH}$.

\section{Protein extraction and iTRAQ labeling}

Total protein was extracted from an equal number $\left(\sim 10^{7}\right)$ of sporulated oocysts of PYS and PRU strains. Following centrifugation at $350 \mathrm{~g}$ for $15 \mathrm{~min}$, oocyst's pellets were suspended in a radioimmunoprecipitation

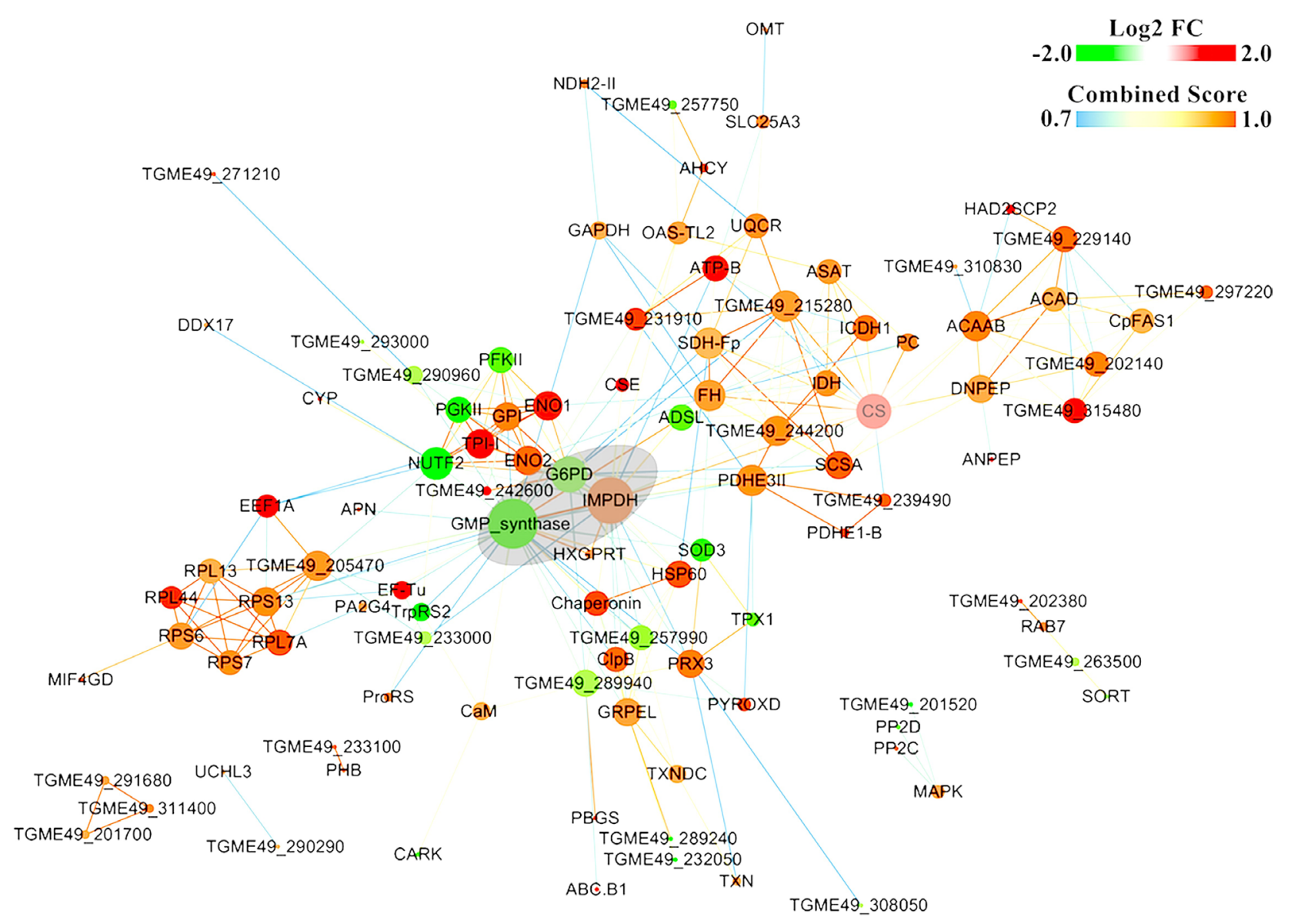

Figure 7: Constructed protein-protein interaction (PPI) networks of the DEPs. PPI networks of the differentially expressed proteins (DEPs) were predicted using STRING and were illustrated using Cytoscape software. Nodes represent DEPs and edges represent interactions between two proteins. Red- and green-colored nodes represent products of increased and decreased DEPs, respectively. The node size indicates high (large) or low (small) degree of interaction. Proteins that are associated to each other are linked by an edge. The color of the edge indicates the combined interaction score (edge score). 
assay (RIPA; $50 \mathrm{mM}$ Tris- $\mathrm{HCl}, 150 \mathrm{mM} \mathrm{NaCl}, 1 \%$ SDS, $0.1 \%$ Trionx-100 and $1 \%$ SDC $\mathrm{pH} 8$ ) buffer supplemented with phenylmethylsulfonyl fluoride (PMSF). The oocyst's suspensions were sonicated on ice and pelleted at 10,625 $\mathrm{g}$ for $20 \mathrm{~min}$ at $4^{\circ} \mathrm{C}$. The supernatant was collected and the protein concentration was measured by BCA assay (QuantiPro ${ }^{\mathrm{TM}}$ BCA Assay Kit, Sigma). About $100 \mu \mathrm{g}$ protein of each sample was reduced, alkylated, and then precipitated by the methanol/chloroform precipitation method. Firstly, $4 \mu \mathrm{L}$ of the Reducing Reagent were added into each sample followed by incubation at $60^{\circ} \mathrm{C}$ for $1 \mathrm{hr}$. Then, the sample was mixed with $2 \mu \mathrm{L}$ CysteineBlocking Reagent and kept at ambient temperature for $10 \mathrm{~min}$. Following rinsing of the $10 \mathrm{KD}$ millipore with $70 \%$ ethanol and deionized water, the protein solution was poured into millipore and centrifuged at $13,400 \mathrm{~g}$ for $20 \mathrm{~min}$. Solution at the bottom of millipore was discarded and the millipore was centrifuged with $100 \mu \mathrm{L} 0.25 \mathrm{M}$ TEAB (tetraethylammonium bicarbonate) $3 \times$ at $13,400 \mathrm{~g}$ for $20 \mathrm{~min}$. The protein pellets were reconstituted in $50 \mu \mathrm{L}$ of $6 \mathrm{M}$ urea $/ 50 \mathrm{mM}$ TEAB with sonication and digested in $2 \%$ trypsin overnight (Promega). Then, peptides were labeled with 8-plex iTRAQ reagents $(113,114,119,121)$ according to the manufacturer's instructions (AB Sciex, USA). About $150 \mu \mathrm{L}$ isopropyl alcohol were added into labeling reagent, and the mixture was added into the samples and incubated for $2 \mathrm{hr}$ at ambient temperature. After termination with deionized water and centrifugation, samples were dried and maintained at $-80^{\circ} \mathrm{C}$ until use.

\section{Strong cation exchange (SCX) fractionation and LC-MS/MS analysis}

The iTRAQ labeled peptides were mixed in equal amounts and fractioned by a high performance liquid chromatography (HPLC) system using Phenomenex columns (Gemini-NX 3u C18 110A; 150*2.00 mm). The dried peptides were dissolved in $100 \mu \mathrm{L}$ buffer $\mathrm{A}$ (20 mM NH4HCO3 in 3\% acetonitrile, pH 10), thoroughly mixed and were then loaded onto Ultremex SCX column. Fractions were collected at a flow rate of $1 \mathrm{~mL} / \mathrm{min}$ with buffer $\mathrm{B}\left(20 \mathrm{mM} \mathrm{NH}_{4} \mathrm{HCO}_{3}, \mathrm{pH} \mathrm{10)}\right.$ at gradients of $0-8 \%$ for $5 \mathrm{~min}$; $8-45 \%$ for $25 \mathrm{~min}$; $45-80 \%$ for $15 \mathrm{~min}$; and $80-100 \%$ for $5 \mathrm{~min}$. Twenty-four fractions were collected and acidified with 50\% Trifluoroacetic acid (TFA). The peptide fractions were dried using vacuum centrifugation and reconstituted in $0.1 \%$ formic acid and $2 \%$ acetonitrile. Then, peptides were centrifuged at $13,447 \mathrm{~g}$ for $20 \mathrm{~min}$ and supernatant was transferred into analytical columns to be identified with an online Q Exactive system (Thermal Scientific). The first grade MS parameters included scan range 350 to $1800 \mathrm{~m} / \mathrm{z}$ with a resolution set at 70,000 with a maximum injection time $40 \mathrm{~ms}$. The second grade MS spectra were acquired in a resolution of 17,500 with $60 \mathrm{~ms}$ maximum injection time and the 20 top precursors for each MS cycle were selected.

\section{Protein quantitation}

Mascot generic format (.mgf) data file transformed from raw MS data by Proteome Discoverer ${ }^{\mathrm{TM}} 1.4$ were queried to $T$. gondii ME49 strain database (http:// www.ToxoDB.org/common/downloads/release-10.0/ TgondiiME49/fasta/data/), which contains 8,322 protein sequences. Proteins were further identified and quantified with ProteinPilot ${ }^{\mathrm{TM}}$ Software 4.5 (AB SCIEX). The parameters for identification and quantification were set as follows: FDR $<0.01$ for identification of peptides and proteins; confidence level of $95 \%$ or unused confidence score larger $>1.3$ for quantification. Proteins with fold

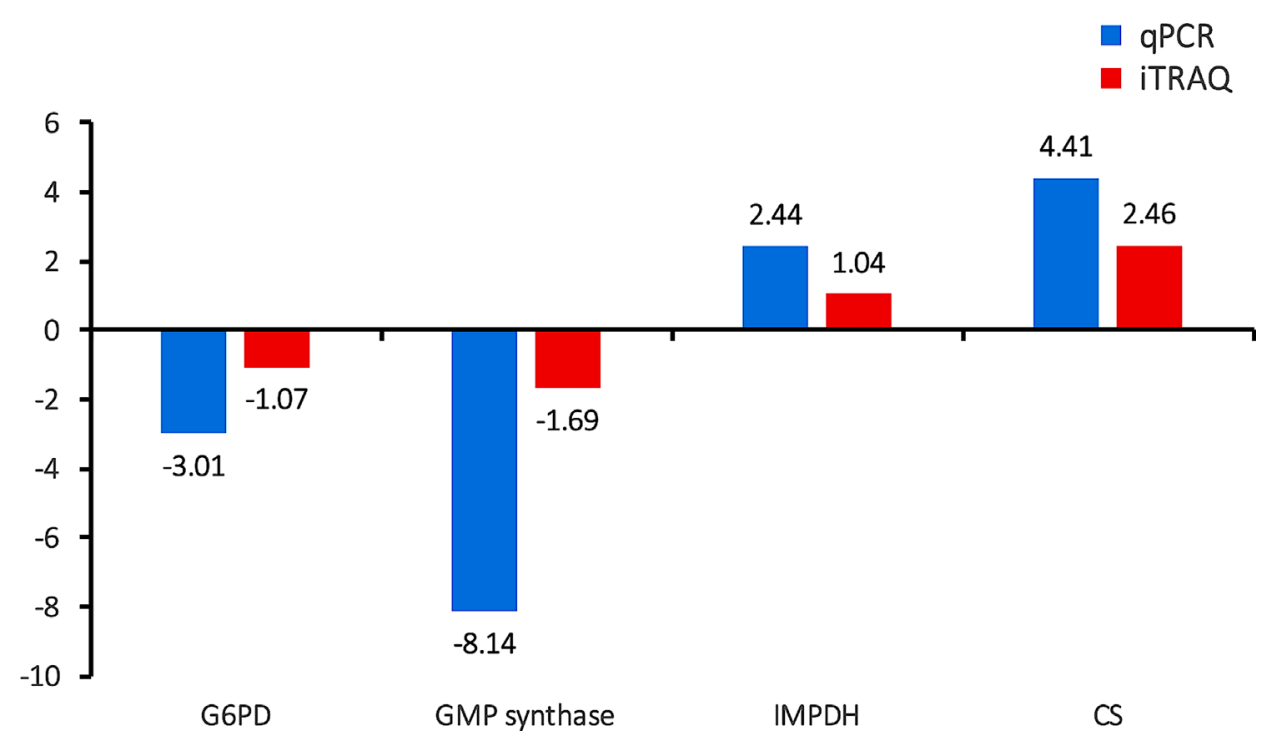

Figure 8: Validation of the relative abundance of the top four hub DEPs by qPCR. $x$-axis shows different DEPs and y-axis shows $\log _{1.5}$ fold change values. 
Table 2: Genes and primers used in qPCR validation

\begin{tabular}{lll}
\hline Gene & Primer name & Primer sequence (5' to $\mathbf{3}^{\prime}$ ) \\
\hline$\beta$-actin & $\beta$-actin-F & CGAGAGGCTGACCAAGGAAC \\
& $\beta$-actin-R & CTGCTGGAAGGTGGAGAGAGA \\
G6PD & G6PD-F & GACGCCTCTGCCGATTTACT \\
& G6PD-R & TCGCTTCCCTTCGCATCT \\
GMP synthase & GMP synthase-F & GGACTTTATGACGGCTGACTGG \\
& GMP synthase-R & TGATGCCCTTGACTTCGTTG \\
IMPDH & IMPDH-F & GAAACTCTGCCTCCTCCTCTC \\
& IMPDH-R & AAGACACCCACAAGCCAAAC \\
CS & CS-F & CCGCAACGCTTAGGAGACTA \\
& CS-R & TACAGGGATTGGCTTCTGGA \\
\hline
\end{tabular}

change ratios $\geq 1.5$ or $\leq 0.67$ were designated as increased or decreased proteins, respectively.

\section{Bioinformatics analysis}

To gain insight into the biological functions of DEPs, gene ontology (GO) classification and enrichment analyses (http://www.geneontology.org) were performed. GO provides a framework for functional annotation and classification of the protein expression data. DEPs were categorized into three main groups: molecular function, biological process and cellular component. Additionally, Kyoto Encyclopedia of Genes and Genomes (KEGG; http://www.genome.jp/kegg/) pathway enrichment analysis was performed to map the potential pathways of the DEPs. Hierarchical clustering analysis and presentation was performed with Cluster 3.0 and java Tree view, respectively. The detection of key proteininteracting ions in the PPI networks was important for the identification of the mechanisms and factors that underpin the virulence of $T$. gondii oocysts. We utilized the Search Tool for the Retrieval of Interacting Genes/Proteins (http:// www.string-db.org/), a database of known and predicted protein interactions, to construct the PPI network and then visualized the distribution characteristics of increased and decreased DEPs in the network with Cytoscape software, version 3.2.0.

\section{Quantitative real-time PCR (q-PCR) validation}

Q-PCR was employed to confirm the gene expression of the identified four hub proteins. TRIzol method was used to extract total RNA from sporulated oocysts of the PYS and PRU strains, respectively, according to the manufacture's instruction (Invitrogen). RNA templates were reverse transcribed to cDNA using a reverse transcription kit (Promega). The $T$. gondii $\beta$-actin was chosen as an endogenous reference gene to normalize all q-PCR data. Q-PCR reactions were performed on the Rotor-Gene Q (QIAGEN) with SYBR Green GoTaq ${ }^{\circledR}$
qPCR Master Mix (Promega). Primers used in this study are shown in Table 2 . The conditions performed in q-PCR cycle were as follows: $95^{\circ} \mathrm{C}$ for 2 min followed by 40 cycles of $95^{\circ} \mathrm{C}$ for $15 \mathrm{~s}, 60^{\circ} \mathrm{C}$ for $30 \mathrm{~s}, 72^{\circ} \mathrm{C}$ for $30 \mathrm{~s}$. The $2^{-\Delta \Delta \mathrm{CT}}$ method was applied to calculate the relative gene expression [31].

\section{Data and materials availability}

All the mass spectrometry data have been submitted to the ProteomeXchange Consortium with identifier PXD005928.

\section{Authors' contributions}

XQZ, DHZ, HME and CXZ conceived and designed the experiments. ZXW, DHZ, CXZ and SH performed the experiments. $\mathrm{CXZ}$ contributed reagents/materials/analysis tools. ZXW and DHZ analyzed the data and wrote the paper. HME and XQZ critically revised the manuscript. All authors read and approved the final version of the manuscript.

\section{ACKNOWLEDGMENTS}

We thank Guangzhou FitGene Biotechnology Co. Ltd. for technical assistance with LC-MS/MS analysis.

\section{CONFLICTS OF INTEREST}

The authors declare no competing financial interests.

\section{FUNDING}

Project financial support was kindly provided by the National Natural Science Foundation of China (Grant Nos. 31230073 and 31172316) and by the Fundamental Research Funds of Chinese Academy of Agricultural Sciences (Grant Nos. 1610312016025, Y2016JC05 and Y2017JC06). 


\section{REFERENCES}

1. Jones JL, Dubey JP. Foodborne toxoplasmosis. J Food Safety. 1991; 55:845-851.

2. Dubey JP. Toxoplasmosis of Animals and Humans. Second edition. Boca Taton, Florida: CRC Press. 2010; 1-313.

3. Tenter AM, Heckeroth AR, Weiss LM. Toxoplasma gondii: from animals to humans. Int J Parasitol. 2000; 30:1217-1258.

4. Petersen E, Dubey JP. Biology of toxoplasmosis (Cambridge University Press, Cambridge, United Kingdom). 2001; $1-42$.

5. Elsheikha HM. Congenital toxoplasmosis: priorities for further health promotion action. Public Health. 2008; 122:335-353.

6. Miller NL, Frenkel JK, Dubey JP. Oral infections with Toxoplasma cysts and oocysts in felines, other mammals, and in birds. J Parasitol. 1972; 58:928-937.

7. Dubey JP. History of the discovery of the life cycle of Toxoplasma gondii. Int J Parasitol. 2009; 39:877-882.

8. Yilmaz SM, Hopkins SH. Effects of different conditions on duration of infectivity of Toxoplasma gondii oocysts. J Parasitol. 1972; 58:938-939.

9. Lindsay DS, Dubey JP. Long-term survival of Toxoplasma gondii sporulated oocysts in seawater. J Parasitol. 2009; 95:1019-1020.

10. Wainwright KE, Lagunas-Solar M, Miller MA, Barr BC, Gardner IA, Pina C, Melli AC, Packham AE, Zeng N, Truong T, Conrad PA. Physical inactivation of Toxoplasma gondii oocysts in water. Appl Environ Microbiol. 2007; 73:5663-5666.

11. Wainwright KE, Miller MA, Barr BC, Gardner IA, Melli AC, Essert T, Packham AE, Truong T, LagunasSolar M, Conrad PA. Chemical inactivation of Toxoplasma gondii oocysts in water. J Parasitol. 2007; 93:925-931.

12. Munoz-Zanzi CA, Fry P, Lesina B, Hill D. Toxoplasma gondii oocyst specific antibodies and source of infection. Emerg Infect Dis. 2010; 16:1591-1593.

13. Robert-Gangneux F, Darde ML. Epidemiology of and diagnostic strategies for toxoplasmosis. Clin Microbiol Rev. 2012; 25:264-296.

14. Xiao J, Yolken RH. Strain hypothesis of Toxoplasma gondii infection on the outcome of human diseases. Acta Physiol (Oxf). 2015; 213:828-845.

15. Saeij JP, Boyle JP, Boothroyd JC. Differences among the three major strains of Toxoplasma gondii and their specific interactions with the infected host. Trends Parasitol. 2005; 21:476-481.

16. Melo MB, Jensen KD, Saeij JP. Toxoplasma gondii effectors are master regulators of the inflammatory response. Trends Parasitol. 2011; 27:487-495.

17. Van Wormer E, Fritz H, Shapiro K, Mazet JA, Conrad PA. Molecules to modeling: Toxoplasma gondii oocysts at the human-animal-environment interface. Comp Immunol Microbiol Infect Dis. 2013; 36:217-231.
18. Jones JL, Dubey JP. Waterborne toxoplasmosis - recent developments. Exp Parasitol. 2010; 124:10-25.

19. Fritz HM, Buchholz KR, Chen X, Durbin-Johnson B, Rocke DM, Conrad PA, Boothroyd JC. Transcriptomic analysis of Toxoplasma development reveals many novel functions and structures specific to sporozoites and oocysts. PLoS One. 2011; 7:e29998.

20. Fritz H, Barr B, Packham A, Melli A, Conrad PA. Methods to produce and safely work with large numbers of Toxoplasma gondii oocysts and bradyzoite cysts. J Microbiol Methods. 2012; 88:47-52.

21. Fritz HM, Bowyer PW, Bogyo M, Conrad PA, Boothroyd JC. Proteomic analysis of fractionated Toxoplasma oocysts reveals clues to their environmental resistance. PLoS One. 2012; 7:e29955.

22. Ferguson DJ, Birch-Andersen A, Siim JC, Hutchison WM. An ultrastructural study on the excystation of the sporozoites of Toxoplasma gondii. Acta Pathol Microbiol Scand B. 1979; 87:277-283.

23. Possenti A, Fratini F, Fantozzi L, Pozio E, Dubey JP, Ponzi M, Pizzi E, Spano F. Global proteomic analysis of the oocyst/sporozoite of Toxoplasma gondii reveals commitment to a host-independent lifestyle. BMC Genomics. 2013; 14:183.

24. Li M, Mo XW, Wang L, Chen H, Luo QL, Wen HQ, Wei W, Zhang AM, Du J, Lu FL, Lun ZR, Shen JL. Phylogeny and virulence divergency analyses of Toxoplasma gondii isolates from China. Parasit Vectors. 2014; 7:64-70.

25. Zhou CX, Zhu XQ, Elsheikha HM, He S, Li Q, Zhou DH, Suo X. Global iTRAQ-based proteomic profiling of Toxoplasma gondii oocysts during sporulation. J Proteomics. 2016; 148:12-9.

26. Dumètre A, Dardé ML. Purification of Toxoplasma gondii oocysts by cesium chloride gradient. J Microbiol Methods. 2004; 56:427-430.

27. Fritz HM, Bowyer PW, Bogyo M, Conrad PA, Boothroyd JC. Proteomic analysis of fractionated Toxoplasma oocysts reveals clues to their environmental resistance. PLoS One. 2012; 7:e29955.

28. Possenti A, Cherchi S, Bertuccini L, Pozio E, Dubey JP, Spano F. Molecular characterization of a novel family of cysteine-rich proteins of Toxoplasma gondii and ultrastructure evidence of oocyst wall localization. Int $\mathrm{J}$ Parasitol. 2010; 40:1639-1649.

29. Hyde JE. Targeting purine and pyrimidine metabolism in human apicomplexan parasites. Curr Drug Targets. 2007; $8: 31-47$.

30. Sullivan WJ Jr, Dixon SE, Li C, Striepen B, Queener SF. IMP dehydrogenase from the protozoan parasite Toxoplasma gondii. Antimicrob Agents Chemother. 2005; 49:2172-2179.

31. Schmittgen TD, Livak KJ. Analyzing real-time PCR data by the comparative C(T) method. Nat Protoc. 2008; 3:1101-8. 